\title{
An improved pharmacometric model for artesunate treatment of falciparum malaria
}

\section{Sompob Saralamba ( $\nabla$ sompob@tropmedres.ac)}

Mahidol Oxford Tropical Medicine Research Unit, Mahidol University

\section{Julie A Simpson}

Centre for Epidemiology and Biostatistics, Melbourne School of Population and Global Health, The University of Melbourne https://orcid.org/0000-0002-2660-2013

\section{Noppon Choosri}

Center of Data Analytics and Knowledge Synthesis for Healthcare, Chiang Mai University

\section{Lisa White}

Big Data Institute, Li Ka Shing Centre for Health Information and Discovery, Nuffield Department of Medicine, University of Oxford https://orcid.org/0000-0002-6523-185X

\section{Wirichada Pan-Ngum}

) Department of Tropical Hygiene, Faculty of Tropical Medicine, Mahidol University https://orcid.org/0000-0002-9839-5359

\section{Arjen M Dondorp}

Mahidol Oxford Tropical Medicine Research Unit, Faculty of Tropical Medicine, Mahidol University

Nicholas J White ( $\square$ nickwdt@tropmedres.ac)

Mahidol Oxford Tropical Medicine Research Unit, Faculty of Tropical Medicine, Mahidol University

\section{Research Article}

Keywords: Plasmodium falciparum, artesunate, artemisinin resistance, mathematical model

Posted Date: February 10th, 2022

DOI: https://doi.org/10.21203/rs.3.rs-1345628/v1

License: (c) (1) This work is licensed under a Creative Commons Attribution 4.0 International License. Read Full License 


\section{Abstract}

Previous mathematical models characterising the pharmacokinetic and pharmacodynamic properties of artemisinins in-vivo have not accounted satisfactorily for observed dose-response relationships. We present a new mathematical model of antimalarial pharmacodynamics which incorporates the hypothesis that parasites enter a transitional unresponsive state after contact with artemisinins, followed either by delayed death or reactivation. The model predictions are consistent with both in vitro and in vivo study results.

\section{Introduction}

The artemisinin derivatives are the cornerstone of current antimalarial therapies. These drugs are highly effective in killing both circulating and sequestered malaria parasites but are eliminated very rapidly from the body. The elimination half-life of the common biologically active metabolite, dihydroartemisinin (DHA), is usually reported as less than one hour. Despite this, in most regimens, the artemisinin derivatives are given only once daily. Simple pharmacometric models, in which parasite killing is a direct function of plasma concentration, predict that with greater exposure to the artemisinins there should be increased parasite killing. This was one of the motivations behind the development of more slowly eliminated synthetic peroxide antimalarial drugs, and was proposed as a solution to the reduced parasite killing associated with artemisinin resistance. However, administering artemisinins more than once daily does not accelerate parasite clearance or improve therapeutic responses ${ }^{1-3}$. It has been suggested that parasite clearance lags behind killing of malaria parasites, and so damaged or dead parasites accumulate relative to live parasites in the circulation ${ }^{4}$. From this hypothesis it was claimed that parasite clearance rate is not a suitable indicator of artemisinin resistance, and that the failure of split dosing to accelerate parasite clearance could still obscure a benefit in cure rates ${ }^{4}$. This was addressed in a metaanalysis of monotherapy trials which showed that split dosing was not associated with higher cure rates 5,6 . Thus, the results of clinical trials do not support this hypothesis, and current pharmacometric models do not explain the observed therapeutic responses satisfactorily.

Taken together these observations are not compatible with simple concentration-dependent malaria parasite killing by artemisinins and current mathematical models do not explain satisfactorily the in-vivo malaria parasite density dynamics following treatment with artemisinin derivatives. Data suggest that potentially reversible parasite injury and delayed parasite stress responses after exposure to the drug might contribute to more complex pharmacodynamics ${ }^{7-15}$. One explanation for the failure of artemisinin monotherapies to achieve $100 \%$ cure rates, even in artemisinin sensitive infections, is the persistence of temporarily drug insensitive or "dormant" parasites $8,11,15,16$.

In the current study we evaluate the hypothesis that, following exposure to artemisinin drugs, parasites can be damaged and rendered temporarily refractory to further injury, and that a fraction of these parasites could recover afterwards. We assessed whether applying this hypothesis in a mathematical 
model could describe satisfactorily parasite clearance dynamics after treatment with artemisinin derivatives.

\section{Results}

The measured DHA drug concentrations of patients given $2 \mathrm{mg} / \mathrm{kg}$ of oral artesunate daily were first analysed to estimate individual specific pharmacokinetic parameters. Supplementary Table S1 and S2 summarises the estimated pharmacokinetic parameters for DHA for the 39 patients. A sequential pharmacokinetic-pharmacodynamic modelling approach was then performed where DHA concentrations were simulated for each individual patient at the corresponding times of the parasitaemia measurements.

\section{Pharmacodynamic model}

The proposed pharmacodynamic model was fitted to the parasite clearance profiles from patients treated with artesunate monotherapy, using a Bayesian hierarchical model ${ }^{17}$. The posterior predictions showed that the model could reproduce the observed parasite dynamics during treatment (Fig. 2 and Supplementary Figure S1 and S3) and the Markov chains of each model parameter converged within the given iterations $(\hat{R}=1)$. The estimated values of the population mean pharmacodynamic parameters are presented in Table 2 .

The clinical study ${ }^{18}$ was conducted in two areas, one where artemisinin resistant parasites were prevalent (Pailin, western Cambodia) and the other where parasites were still sensitive to artemisinin (Wang Pha, western border of Thailand). The population means of estimated recovery and death rates of each parasite asexual stage showed that parasite recovery rates were slightly different between stages and sites. Recovery rates from Pailin, western Cambodia were slightly lower than the recovery rates from Wang Pha, western Thailand. As expected the estimated death rates of ring stage parasites in Pailin were substantially (over ten fold) lower than those of Wang Pha (Table 2). The estimated death rates for trophozoite and schizont stage parasites were not different between the locations. This is supported by concomitant standard 48 hour in-vitro susceptibility studies which showed little difference in artesunate susceptibility between the sites ${ }^{18}$. The box-and-whisker plots of recovery and death rates for ring, trophozoite and schizont stage parasites compared between the clinical sites are shown in Fig. 3. The population means of the recovery lag-times for damaged parasites to recover to normal state were 2.42 (0.04-7.00) hours in Wang Pha and 2.01 (0.04-7.32) hours in Pailin.

\section{Split dose simulation}

For the split-dose simulation, the DHA concentration profiles were incorporated in the model every 12 hours for 7 days (i.e. a dosing regimen of $2 \mathrm{mg} / \mathrm{kg}$ oral artesunate every 12 hours for 7 days) and parasite clearance was determined from the corresponding simulated parasitaemia profiles (Fig. 4). Figure 5 compares the parasite clearance profiles of the daily and split dose (every 12 hours) artesunate dosing regimens. These distributions of parasite clearance show that, within the same clinical site, the 
median and spread of parasite clearance following daily and 12 hourly drug administration are not significantly different (Fig. 6 and Table S3).

\section{Discussion}

Mathematical models describing the pharmacometric properties of the artemisinin antimalarials have assumed a simple and direct relationship between drug exposure and malaria parasite killing. Because these drugs are eliminated very rapidly these models naturally predicted that more sustained exposure, created by slowing drug clearance, administering by constant infusion or by frequent dosing, would enhance parasite clearance, and thus improve therapeutic responses ${ }^{4,19,20}$. This was proposed as a solution to the challenge posed by artemisinin resistant $P$. falciparum infections. However, clinical studies did not confirm these predictions ${ }^{3}$ and there was no significant advantage in terms of parasite clearance or cure rate from giving the artemisinin derivatives more than once daily despite their rapid elimination. The simple models thus appear to be wrong.

Artemisinin resistance in P. falciparum is characterised by a reduced parasite clearance rate in vivo ${ }^{18}$. This reflects reduced susceptibility of the circulating ring stage parasites to the drug ${ }^{21}$. Artemisinin resistance in field isolates is associated with mutations in the propeller region of the Pfkelch gene on chromosome 13 (K13). This causal association has been confirmed in transfection studies, although the contribution of other genes (often described collectively as the genetic background) is substantial 22 . Artemisinin resistance is thought to involve altered parasite cellular responses rather than receptor or transporter alterations, but the exact mechanism remains unclear ${ }^{23}$. As the more mature stages of $\mathrm{K} 13$ mutant $P$. falciparum isolates remain sensitive to the artemisinin derivatives the drugs do remain efficacious in clinical practice, but they kill less parasites per asexual cycle and so the therapeutic responses are diminished. Longer exposures from longer courses over three or four asexual cycles improve therapeutic responses in artemisinin resistant infections, but giving the drugs more frequently than once daily does not ${ }^{1-3}$.

In the present study, a new mathematical model expanding the earlier simpler models $(20,21)$ was evaluated. This model was constructed to account for the dose-response relationship and could explain the lack of additional effect by more frequent administration. The proposed model incorporated the hypothesis that, in the presence of artemisinins, some parasites are injured, stop growing and become temporarily insensitive to the drug. These parasites can, however, recover and return to their normal, drugsensitive state. Whether this unresponsive ("injury") state causing a temporary arrest in development is the same or a similar process as the well described "dormancy" phenomenon has not been specified, and both processes can explain recrudescences following standard treatments is not specified ${ }^{9}$. The model was fitted to parasite count data obtained from patients in western Cambodia (where artemisinin resistance was prevalent) and western Thailand (before the main emergence of artemisinin resistance there) ${ }^{18}$. The model captured satisfactorily the dynamics of $P$. falciparum parasites in patients receiving artesunate monotherapy every 24 hours for 7 days. As reported previously ${ }^{19}$ the major difference 
between parasites from Pailin, which were artemisinin resistant, and those from Wang Pha, which were sensitive, was the more than ten fold estimated reduction in ring stage killing. Trophozoite stage killing was similar and schizont killing was reduced by about $45 \%$. Recovery rates were slightly but not significantly different between the two sites. This suggests, if this hypothesis is correct, that recovery rates from parasite injury are not greatly affected by the mechanisms involved in artemisinin resistance.

The lower predicted death rates of the asexual ring stage parasites in the artemisinin resistant infections 19 is supported by extensive experimental investigations ${ }^{7-12,21,24,25}$ and the strong correlation between specific ring stage in-vitro susceptibility evaluations, K13 mutations and slow parasite clearance. The unresponsive ("injury") state postulated in this model suggested that the parasites were in a damaged state between 2 and 50 hours prior to dying. The estimated parasite recovery rate from this model was about 1 in 740-850 unresponsive parasites per 24 hours. The estimate of the recovery lag-time was 22.4 hours suggesting that the parasites became damaged or dormant shortly after being exposed to the drug.

This model was developed to account for the failure of frequent dosing regimens to accelerate parasite clearance or enhance cure rates in artemisinin containing antimalarial drug regimens. It did this satisfactorily but there are several limitations to this modelling exercise. Many of the pharmacodynamic parameter values in this model have not been measured directly so the system is unidentifiable. It has also simplified the complex relationship between parasite stage of development, and time and intensity of drug exposure, and assumed homogeneous parasite stage distributions and multiplication and elimination kinetics. Although the model can reproduce the observed data, this does not mean that it has explained the underlying biology (i.e. the model may not be correct). Nevertheless, it does represent a relatively simple hypothesis that is consistent with observations and is testable. But there may well be other hypotheses whose models would fit equally well with the data. We propose that, as a minimum, such models should be capable of reproducing both delayed clearance and the unchanged clearance rates under frequent dosing in order to be consistent with observed data.

In conclusion, a new within-host pharmacometric model is proposed, which supports the hypothesis that parasites enter a temporary drug refractory injury state after contact with artemisinin antimalarials, which is followed by delayed death or reactivation. The model fitted the observed sequential parasitaemia data from patients with artemisinin resistant and sensitive $P$. falciparum infections and confirmed the known dose-response relationship for reduced ring stage activity in artemisinin resistant infections.

\section{Methods}

\section{Parasite clearance data and pharmacokinetic data}

We re-evaluated data used to generate an earlier simpler mathematical model in which there was a direct concentration-dependent malaria parasite killing (18). The data used in this evaluation were serial parasite count data (asexual parasites) and drug concentration data (dihydroartemisinin (DHA)) from 20 
patients from Pailin, western Cambodia and 19 patients from Wang Pha, western Thailand. These patients were treated with artesunate monotherapy as part of a clinical trial to investigate artemisinin resistance conducted during 2007 and 2008 by Dondorp et al. ${ }^{18}$. Patients received $2 \mathrm{mg} / \mathrm{kg}$ oral artesunate every $24 \mathrm{~h}$ for 7 days. Parasite counts were determined by microscopy at $0,4,8$ and $12 \mathrm{~h}$, and then every $6 \mathrm{~h}$ until two consecutive negative slides were recorded. DHA concentrations were measured at $0,0.25,0.5,1,1.5,2,3,4,5,6,8$ and $12 \mathrm{~h}$ following the first dose.

\section{Mathematical model}

The base model is that of White et al. describing the within-patient dynamics of $P$. falciparum parasites $19,25,26$. The age distribution of the parasites at any time before treatment was assumed to be unimodal and Gaussian. The asexual life cycle was assumed to be 48 hours. This distribution shifts to the right as parasites become older. The infection multiplication factor is the population average number of merozoites which successfully infect new red blood cells (RBCs) per one infected RBC. During the expansion phase of the infection this is a positive number, limited by the average number of merozoites per schizont. Time in the model was discretised and fixed at 1 hour intervals. Asexual $P$. falciparum parasites were therefore divided into 48 individual age-groups, from 1 to 48 hours. As the parasites become older by 1 hour the number of parasites moves to the next age-group, until at age 47-48 hours at schizogony, after which they become ring stage parasites with aged $0-1$ hour at a density defined by the multiplication factor.

\section{Antimalarial pharmacodynamics}

Antimalarial drugs kill malaria parasites, but the pharmacodynamic effect depends both on the plasma concentration of drug and the susceptibility of the predominant stage of parasite development. When the antimalarial drug (in this case an artemisinin derivative) is present, a fraction of parasites at each susceptible stage will be damaged and some of these die (and subsequently "pitted" from the infected $\mathrm{RBC}$ by the spleen). It is hypothesised that a fraction of the remainder of the damaged parasites can recover potentially, but their development is arrested, during which period the parasites are refractory to further injury by the antimalarial drug. In the presence of the drug, the fraction of the damaged parasites ( $\mathrm{f}(t))$ at time $t$ is calculated by:

$$
\mathrm{f}(t)=\frac{E_{m} c(t)^{\gamma}}{c(t)^{\gamma}+E c_{50}^{\gamma}}
$$

1

where $E_{m}$ is the maximum extent (\%) that the parasites can be damaged by the drug, $c(t)$ is the concentration of DHA at time $t$ hours, $E C_{50}$ is the concentration that gives $50 \%$ of the damage effect, and yis the slope constant. Damaged parasites that recover after a lag time return to their pre-treatment stage, with normal aging, multiplication and pre-treatment drug susceptibility. Parasites die at a rate of $\nu$ (/hour) and surviving parasites will produce new merozoites. The fraction of damaged parasites that recover to their pre-treatment normal state at time $t, K(t)$, is calculated from 


$$
K(t)=\frac{r_{m}}{1+\exp (-\mathrm{f}(t) \times(t-\operatorname{lag}))}
$$

\section{2}

where $r_{m}$ is the maximum fraction of the damaged parasites that can recover, $\mathrm{f}(t)$ is the fraction of the damaged parasites at time $t$, and lag is the recovery lag time. These recovery and death fractions are assumed to depend on the stage of development of the parasites. The susceptibility of malaria parasites to all antimalarial drugs is strongly dependent on their stage of development. For simplicity we divide the parasites into three developmental stages; rings ( $0-26$ hours), trophozoites (27-38 hours) and schizonts (39-48 hours). Laboratory studies suggest that young ring stages are most likely to enter a state of dormancy, particularly following exposure to artemisinin derivatives $8,10,11,15$. A diagram of the model is shown in Fig. 1. The plasma concentration of DHA, $c(t)$, following each oral dose was modelled as follows:

$$
c(t)=\left\{\begin{array}{l}
\left(\frac{c_{m} t}{t_{m}}\right), t \leq t_{m} \\
c_{m} e^{-k t}, t>t_{m}
\end{array}\right.
$$

3

where $t_{m}$ is the time of maximum concentration, $c_{m}$ is the maximum concentration, and $k$ is the firstorder elimination rate constant of the drug. DHA elimination is well characterised by a a single rate constant 27,28 .

The pharmacodynamic model output was the number of circulating parasites calculated from the number of parasites at each age multiplied by the observable probability function of the parasites at different ages, as previously proposed by Saralamba et al. ${ }^{19}$. See the supplementary information for the Stan ${ }^{17}$ code of the model.

\section{Statistical analysis}

The concentration model in Eq. (3) was fitted to the DHA concentration profile of each patient separately following their first oral dose of artesunate $(2 \mathrm{mg} / \mathrm{kg})$. This fitting was done in Wolfram Mathematica ${ }^{29}$. The estimated concentration parameters $\left(t_{m}, c_{m^{\prime}}, k\right)$ were used as the input for generating the DHA plasma concentration at any time $t$ following each dose during treatment for each individual patient.

The proposed pharmacodynamic model was fitted to the observed parasite count data obtained during artesunate monotherapy using a Bayesian hierarchical model approach for estimating the model parameters at both individual and population levels. The model parameters were transformed and reparameterised using the method proposed by Lesaffre et $a^{30}{ }^{30}$. In this the pharmacodynamic model 
parameters $\left(\theta_{\text {ind }}\right)$ for each individual and their lower $\left(b_{L}\right)$ and upper $\left(b_{U}\right)$ bounds were transformed to the unbounded parameters $\phi_{\text {ind }}=\ln \left(\frac{\theta_{\text {ind }}-b_{L}}{b_{U}-\theta_{\text {ind }}}\right)$. And the unbounded population means were transformed from $\phi_{p o p}=\ln \left(\frac{\theta_{p o p}-b_{L}}{b_{U}-\theta_{p o p}}\right)$, where $\theta_{p o p}$ is the population mean of $\theta_{\text {ind. }}$ In the sampling process, these individual parameters $\phi_{\text {ind }}$ were reparameterised to be $\phi_{\text {ind }}=\phi_{\text {pop }}+\omega_{p o p} L \eta$ where $\omega_{\text {pop }}$ - the standard deviation (SD), $L$ - the lower Cholesky factor and $\eta \sim \mathcal{N}(0,1)$.

The fitting was implemented in Stan using the Hamiltonian Monte Carlo (HMC) method ${ }^{17}$. The likelihood function was derived assuming that log10 transformed of each observed parasite density measurement ( $\left.\Phi_{\mathrm{i}}\right)$ at time $i$ was sampled from a normal distribution, of which the mean was the model output $\left(\mathrm{M}_{\mathrm{i}}\right)$ for that observed time $i$ and its standard deviation was $\varrho$. That is, the likelihood can be written as:

$$
\log _{10} \Phi_{\mathrm{i}} \sim \operatorname{Normal}\left(\mathcal{M}_{i}, \varrho\right)
$$

The first 1,000 parameter values sampled for each chain were discarded as burn-in and the subsequent 1,000 samples from each chain $(n=3)$ were used to estimate the posterior distributions and also the posterior predictive checks. The convergence of the chains of each parameter was assessed using trace plots and the $\hat{R}$ statistic ${ }^{31}$. The prior distribution of each model parameter was the uniform distribution with the boundaries as shown in Table 1. The Stan and C\# code of the model can be downloaded from https://github.com/slphyx/DamagedParasites.

\section{Split-dose simulation}

To predict the effect of administering artesunate to patients every 12 hours for 7 days, the models were re-run using parameter values sampled from the posterior distributions estimated from fitting the model to parasite clearance profiles following artesunate monotherapy once daily, except that drug concentration profiles were changed from every 24 hours to every 12 hours for 7 days. For each hypothetical patient, parasite clearance times were calculated from the parasite versus time profiles which were sampled from the fitted hourly profiles of each patient. Here the parasite clearance time was defined as the time from first dose of artesunate to the time that the simulated circulating parasite count was below the detection limit $(\mathrm{L})$, which differed between the hypothetical patients and was estimated from:

$$
\mathrm{L} \approx 1 \times 80,000 \times \text { weight }
$$

5

where $l$ is the lowest parasite count number (parasites $/ \mu \mathrm{L}$ ), and weight is the recorded body weight of the patient $(\mathrm{kg})^{19}$. All patients were assumed to have a total blood volume $80 \mathrm{~mL} / \mathrm{kg}$. 
To test whether the model could reproduce the trial results from Das et al. ${ }^{3}$, which showed no improvement in parasite clearance time whether patients were given $2 \mathrm{mg} / \mathrm{kg}$ artesunate every 12 or 24 hours for 7 days, clearance times predicted by simulating both dose regimens were compared visually.

\section{Declarations}

\section{Acknowledgements}

This research was funded in part by the Wellcome Trust [Grant number 220211].

\section{Competing interests}

The authors declare no competing interests.

\section{References}

1. ter Kuile, F. O. et al. High-dose mefloquine in the treatment of multidrug-resistant falciparum malaria. $J$ Infect Dis 166, 1393-1400 (1992).

2. Ursing, J., Rombo, L., Bergqvist, Y., Rodrigues, A. \& Kofoed, P. E. High-Dose Chloroquine for Treatment of Chloroquine-Resistant Plasmodium falciparum Malaria. J Infect Dis 213, 1315-1321, doi:10.1093/infdis/jiv590 (2016).

3. Das, D. et al. Effect of high-dose or split-dose artesunate on parasite clearance in artemisininresistant falciparum malaria. Clin Infect Dis 56, e48-58, doi:10.1093/cid/cis958 (2013).

4. Hastings, I. M., Kay, K. \& Hodel, E. M. How Robust Are Malaria Parasite Clearance Rates as Indicators of Drug Effectiveness and Resistance? Antimicrob Agents Chemother 59, 6428-6436, doi:10.1128/AAC.00481-15 (2015).

5. White, N. J., Watson, J. \& Ashley, E. A. Split dosing of artemisinins does not improve antimalarial therapeutic efficacy. Scientific reports 7, 12132, doi:10.1038/s41598-017-12483-4 (2017).

6. White, N. J. Malaria parasite clearance. Malar J 16, 88, doi:10.1186/s12936-017-1731-1 (2017).

7. Nosten, F. Waking the sleeping beauty. J Infect Dis 202, 1300-1301, doi:10.1086/656478 (2010).

8. Teuscher, F. et al. Artemisinin-induced dormancy in plasmodium falciparum: duration, recovery rates, and implications in treatment failure. J Infect Dis 202, 1362-1368, doi:10.1086/656476 (2010).

9. Codd, A., Teuscher, F., Kyle, D. E., Cheng, Q. \& Gatton, M. L. Artemisinin-induced parasite dormancy: a plausible mechanism for treatment failure. Malar J 10, 56, doi:10.1186/1475-2875-10-56 (2011).

10. Cheng, Q., Kyle, D. E. \& Gatton, M. L. Artemisinin resistance in Plasmodium falciparum: A process linked to dormancy? Int J Parasitol Drugs Drug Resist 2, 249-255, doi:10.1016/j.ijpddr.2012.01.001 (2012).

11. Witkowski, B. et al. Increased tolerance to artemisinin in Plasmodium falciparum is mediated by a quiescence mechanism. Antimicrob Agents Chemother 54, 1872-1877, doi:10.1128/AAC.01636-09 (2010). 
12. Klonis, N. et al. Altered temporal response of malaria parasites determines differential sensitivity to artemisinin. Proc Natl Acad Sci U S A 110, 5157-5162, doi:10.1073/pnas.1217452110 (2013).

13. Dogovski, C. et al. Targeting the cell stress response of Plasmodium falciparum to overcome artemisinin resistance. PLoS biology 13, e1002132, doi:10.1371/journal.pbio.1002132 (2015).

14. Cao, P. et al. A Dynamic Stress Model Explains the Delayed Drug Effect in Artemisinin Treatment of Plasmodium falciparum. Antimicrob Agents Chemother 61, doi:10.1128/AAC.00618-17 (2017).

15. Peatey, C. et al. Dormant Plasmodium falciparum Parasites in Human Infections Following Artesunate Therapy. J Infect Dis 223, 1631-1638, doi:10.1093/infdis/jiaa562 (2021).

16. Khoury, D. S. et al. Malaria Parasite Clearance: What Are We Really Measuring? Trends Parasitol 36, 413-426, doi:10.1016/j.pt.2020.02.005 (2020).

17. Carpenter, B. et al. Stan: A Probabilistic Programming Language. Journal of Statistical Software 76, doi:10.18637/jss.v076.i01 (2017).

18. Dondorp, A. M. et al. Artemisinin resistance in Plasmodium falciparum malaria. N Engl J Med 361, 455-467, doi:10.1056/NEJMoa0808859 (2009).

19. Saralamba, S. et al. Intrahost modeling of artemisinin resistance in Plasmodium falciparum. Proc Natl Acad Sci U S A 108, 397-402, doi:10.1073/pnas.1006113108 (2011).

20. Kay, K., Hodel, E. M. \& Hastings, I. M. Altering Antimalarial Drug Regimens May Dramatically Enhance and Restore Drug Effectiveness. Antimicrob Agents Chemother 59, 6419-6427, doi:10.1128/AAC.00482-15 (2015).

21. Witkowski, B. et al. Reduced artemisinin susceptibility of Plasmodium falciparum ring stages in western Cambodia. Antimicrob Agents Chemother 57, 914-923, doi:10.1128/AAC.01868-12 (2013).

22. Stokes, B. H. et al. Plasmodium falciparum K13 mutations in Africa and Asia impact artemisinin resistance and parasite fitness. eLife 10, doi:10.7554/eLife.66277 (2021).

23. Ariey, F. et al. A molecular marker of artemisinin-resistant Plasmodium falciparum malaria. Nature 505, 50-55, doi:10.1038/nature12876 (2014).

24. Hott, A., Tucker, M. S., Casandra, D., Sparks, K. \& Kyle, D. E. Fitness of artemisinin-resistant Plasmodium falciparum in vitro. J Antimicrob Chemother 70, 2787-2796, doi:10.1093/jac/dkv199 (2015).

25. Hoshen, M. B., Na-Bangchang, K., Stein, W. D. \& Ginsburg, H. Mathematical modelling of the chemotherapy of Plasmodium falciparum malaria with artesunate: postulation of 'dormancy', a partial cytostatic effect of the drug, and its implication for treatment regimens. Parasitology 121 (Pt 3), 237-246 (2000).

26. White, N. J., Chapman, D. \& Watt, G. The effects of multiplication and synchronicity on the vascular distribution of parasites in falciparum malaria. Trans R Soc Trop Med Hyg 86, 590-597 (1992).

27. Saunders, D. et al. Pharmacokinetics and pharmacodynamics of oral artesunate monotherapy in patients with uncomplicated Plasmodium falciparum malaria in western Cambodia. Antimicrob Agents Chemother 56, 5484-5493, doi:10.1128/AAC.00044-12 (2012). 
28. Lohy Das, J. et al. Population Pharmacokinetic and Pharmacodynamic Modeling of Artemisinin Resistance in Southeast Asia. Aaps J 19, 1842-1854, doi:10.1208/s12248-017-0141-1 (2017).

29. Mathematica v. 12.3.1 (Wolfram Research, Inc., Champaign, Illinois, 2021).

30. Lesaffre, E., Rizopoulos, D. \& Tsonaka, R. The logistic transform for bounded outcome scores. Biostatistics 8, 72-85, doi:10.1093/biostatistics/kxj034 (2007).

31. Gelman, A. \& Rubin, D. B. Inference from Iterative Simulation Using Multiple Sequences. Statistical Science 7, 457-472, doi:10.1214/ss/1177011136 (1992).

\section{Tables}

Table 1

Parameter definitions for the artesunate pharmacodynamic model and chosen prior distributions

\begin{tabular}{|c|c|c|c|}
\hline Parameter description & Symbol & $\begin{array}{l}\text { Prior } \\
\text { distribution }\end{array}$ & Unit \\
\hline $\begin{array}{l}\text { Initial number of parasites in the body on admission } \\
\text { (log } 10 \text { scale) }\end{array}$ & $N_{0}$ & $\mathrm{U}(8,13)$ & parasites \\
\hline Mean age of parasites on admission & $\mu$ & $\mathrm{U}(1,48)$ & hours \\
\hline $\begin{array}{l}\text { Standard deviation of the age of parasites on } \\
\text { admission }\end{array}$ & $\sigma$ & $\mathrm{U}(1,48)$ & hours \\
\hline Parasite multiplication factor per cycle & $p m f$ & $\mathrm{U}(1,30)$ & $\begin{array}{l}\text { parasites per } 48 \\
\text { hours }\end{array}$ \\
\hline $\begin{array}{l}\text { Maximum extent (\%) that the parasites can be } \\
\text { damaged by the drug }\end{array}$ & $E_{m}$ & $\mathrm{U}(50,99.99)$ & $\begin{array}{l}\text { percentage per } \\
\text { hour }\end{array}$ \\
\hline $\begin{array}{l}\text { Plasma concentration of DHA that gives } 50 \% \text { of the } \\
\text { damaged effect }\end{array}$ & $E C_{50}$ & $\mathrm{U}(5,100)$ & $\mathrm{ng} / \mathrm{ml}$ \\
\hline Slope of the parasite damage response curve & $\gamma$ & $\mathrm{U}(1.5,9.5)$ & \\
\hline Recovery rate of each asexual stage* & K & $\mathrm{U}(0-0.001)$ & /hour \\
\hline Death rate of each asexual stage* & $\nu$ & $\mathrm{U}(0,1)$ & /hour \\
\hline Lag time & $\operatorname{lag}$ & $\mathrm{U}(0,48)$ & hours \\
\hline
\end{tabular}


Table 2

Posterior summaries for the population mean pharmacodynamic parameters calculated from 1000 draws from the posterior distribution

\begin{tabular}{|c|c|c|}
\hline & Pailin & Wang Pha \\
\hline \multirow[t]{2}{*}{ Parameter } & Posterior Median & Posterior Median \\
\hline & (95\% Credible Interval) & (95\% Credible Interval) \\
\hline $\begin{array}{l}\text { Initial number of parasites } \\
\text { in the body on admission } \\
\text { (log } 10 \text { scale) }\end{array}$ & $12.13(11.76,12.49)$ & $11.64(11.29,11.94)$ \\
\hline $\begin{array}{l}\text { Mean age of parasites on } \\
\text { admission (hours) }\end{array}$ & $18.98(15.77,22.24)$ & $16.23(14.06,18.31)$ \\
\hline $\begin{array}{l}\text { Standard deviation of the } \\
\text { age of parasites on } \\
\text { admission (hours) }\end{array}$ & $8.43(7.36,10.14)$ & $5.82(4.73,6.62)$ \\
\hline $\begin{array}{l}\text { Parasite multiplication } \\
\text { factor (/48 hours) }\end{array}$ & $3.54(1.042,19.93)$ & $6.27(1.26,23.10)$ \\
\hline $\begin{array}{l}\text { Slope of the damage } \\
\text { response curve }\end{array}$ & $6.11(1.56,9.89)$ & $6.52(1.99,9.75)$ \\
\hline $\begin{array}{l}\text { Plasma concentration of } \\
\text { DHA that gives } 50 \% \text { of the } \\
\text { damage effect }(\mathrm{ng} / \mathrm{ml})\end{array}$ & $43.11(5.30,85.46)$ & $54.26(6.18,95.65)$ \\
\hline Death rate of Rings (/hour) & $0.0267(0.0024,0.0822)$ & $0.2890(0.1188,0.4018)$ \\
\hline $\begin{array}{l}\text { Death rate of Trophozoites } \\
\text { (/hour) }\end{array}$ & $0.3429(0.2250,0.4616)$ & $0.3811(0.2364,0.4649)$ \\
\hline $\begin{array}{l}\text { Death rate of Schizonts } \\
\text { (/hour) }\end{array}$ & $0.2187(0.0283,0.4494)$ & $0.3866(0.1070,0.4963)$ \\
\hline \multirow{2}{*}{$\begin{array}{l}\text { Recovery rate of Rings } \\
\text { (/hour) }\end{array}$} & $4.786 \times 10^{-5}$ & $5.499 \times 10^{-5}$ \\
\hline & $\left(2.779 \times 10^{-7}, 9.629 \times 10^{-5}\right)$ & $\left(3.261 \times 10^{-6}, 9.482 \times 10^{-5}\right)$ \\
\hline \multirow{2}{*}{$\begin{array}{l}\text { Recovery rate of } \\
\text { Trophozoites (/hour) }\end{array}$} & $4.816 \times 10^{-5}$ & $5.543 \times 10^{-5}$ \\
\hline & $\left(1.793 \times 10^{-6}, 9.593 \times 10^{-5}\right)$ & $\left(1.642 \times 10^{-6}, 9.667 \times 10^{-5}\right)$ \\
\hline \multirow{2}{*}{$\begin{array}{l}\text { Recovery rate of Schizonts } \\
\text { (/hour) }\end{array}$} & $4.983 \times 10^{-5}$ & $5.696 \times 10^{-5}$ \\
\hline & $\left(3.893 \times 10^{-6}, 9.797 \times 10^{-5}\right)$ & $\left(3.186 \times 10^{-6}, 9.629 \times 10^{-5}\right)$ \\
\hline Lag time (hours) & $2.01(0.04,7.32)$ & $2.42(0.04,7.01)$ \\
\hline
\end{tabular}

\section{Figures}




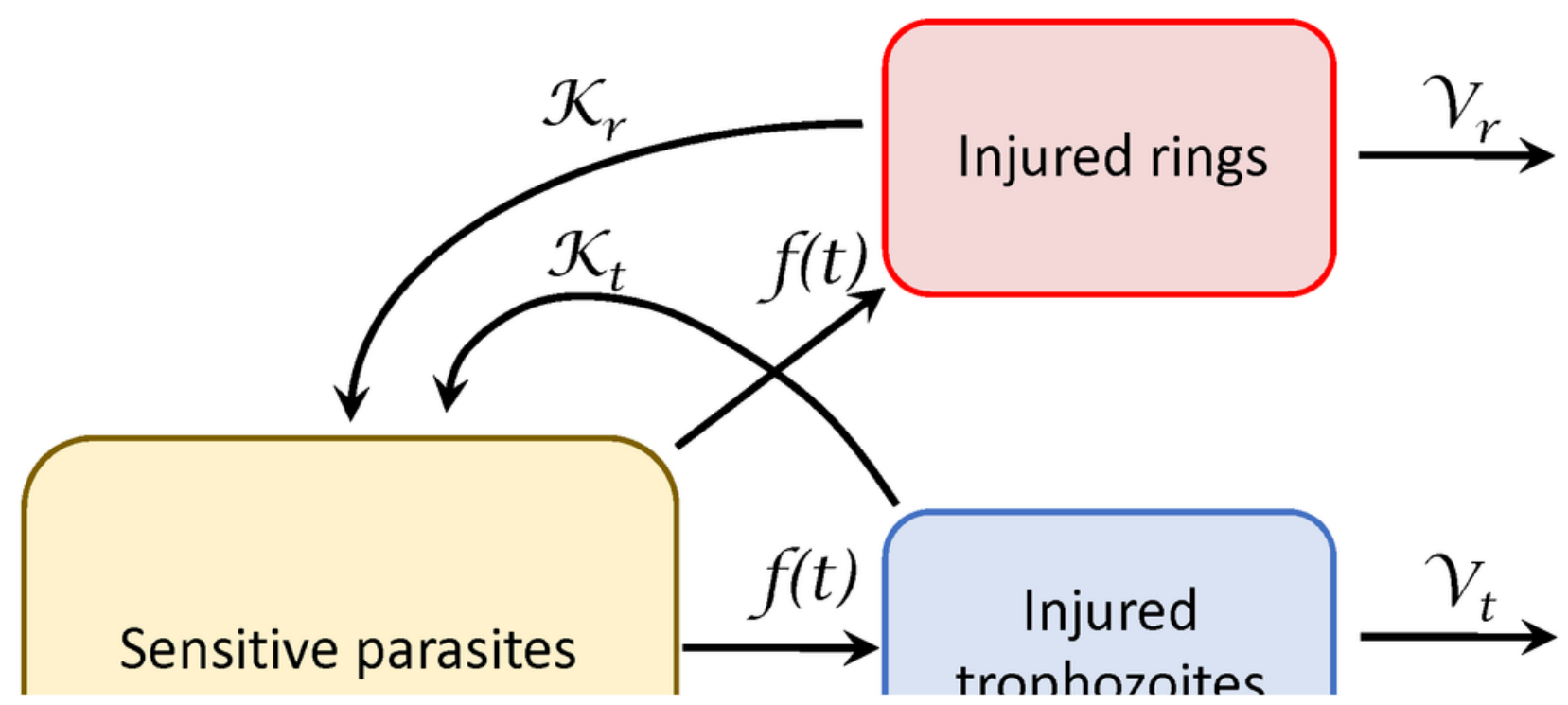

\section{Figure 1}

A diagram of the proposed model, where $\mathrm{f}(\mathrm{t})$ is the fraction of sensitive parasites at time $t$ that can be damaged, $\mathrm{K}$ is the recovery rate of each asexual stage, and $\mathrm{v}$ is the death rate of each asexual stage.

\section{Figure 2}

Example results from fitting the pharmacodynamic model to the parasite count data from patients who received artesunate monotherapy in (A) Wang Pha, Thailand, and (B) Pailin, Cambodia. In each plot, the blue dots represent the observed data and the grey line represents the median of the model outputs. The light-grey shaded area represents the $95 \%$ credible intervals (Cls). See Supplementary information for plots of parasitaemia profiles for all 39 patients. 


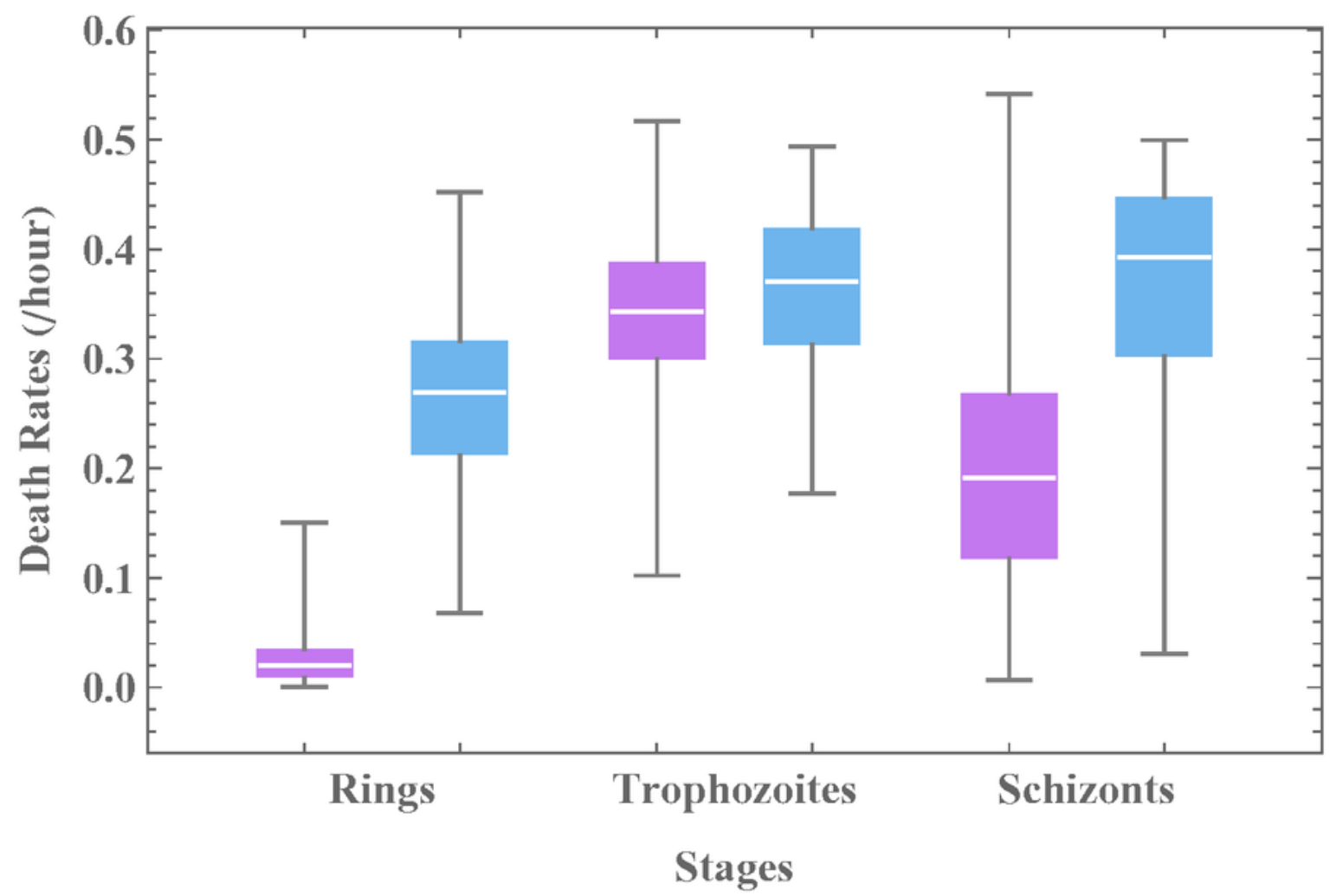

$\square$ Pailin
$\square$ Wang Pha

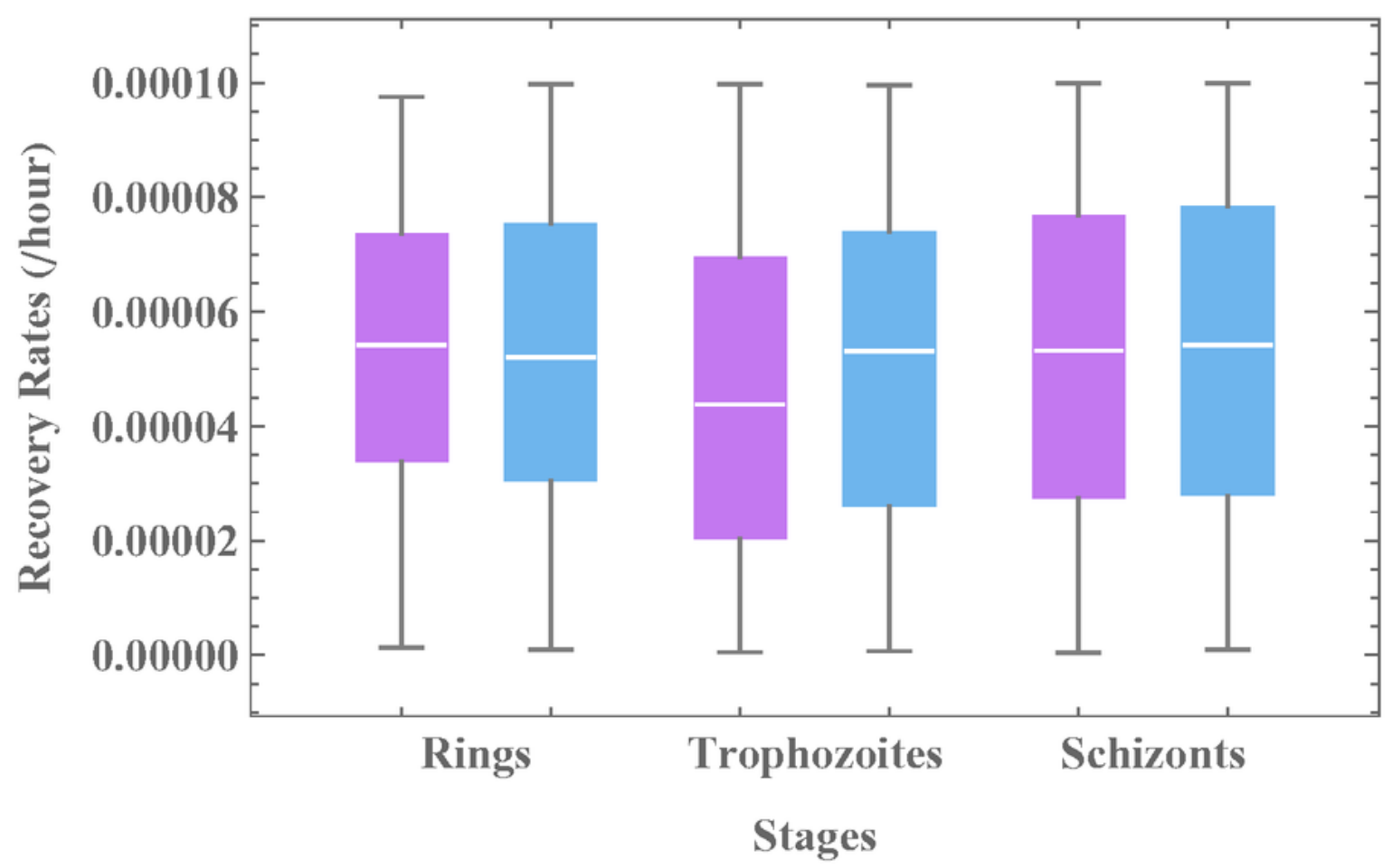

$\square$ Pailin

$\square$ Wang Pha

Figure 3 
Box-and-whisker plots generated from the posterior distributions of estimated population means of the asexual stage specific death rates (top) and recovery rates (bottom) for each study site (purple = Pailin, western Cambodia; blue = Wang Pha, western Thailand).

\section{Figure 4}

Example results from the dose-splitting simulation in (A) Wang Pha, Thailand and (B) Pailin, Cambodia. The black dots represent the observed data, the purple lines represent the median of the model outputs for taking the drug every 24 hours and the green lines represent the model outputs for taking the drug every 12 hours. See the Supplementary information for plots of parasitaemia profiles for all 39 patients.

\section{Figure 5}

Comparison of parasite clearance time (hours) derived from simulated parasitaemia profiles of hypothetical patients receiving $2 \mathrm{mg} / \mathrm{kg}$ of artesunate every 24 hours and every 12 hours for 7 days from (A) Wang Pha, western Thailand and (B) Pailin, wester Cambodian.

\section{Figure 6}

Comparison of distribution of parasite clearance times (hours) derived from simulated parasitaemia profiles for a single dose of artesunate every 24 hours (thick lines) versus every 12 hours (dashed lines) for 7 days from Pailin, western Cambodian (Purple) and Wang Pha, western Thailand (Blue).

\section{Supplementary Files}

This is a list of supplementary files associated with this preprint. Click to download.

- Supplementary.pdf 